\title{
The Level of Bone Metabolism Indexes in Patients with Chronic Obstructive Pulmonary Disease and Its Clinical Significance
}

\author{
XIAOFEN HUANG AND XIAOQIU WU*
}

Department of Respiratory, People's Hospital of Chongzuo, Chongzuo City, Guangxi Zhuang Autonomous 532200, China

Huang et al.: Chronic Obstructive Pulmonary Disease and Its Clinical Significance

\begin{abstract}
To determine the level of bone metabolism indexes in patients with chronic obstructive pulmonary disease and its clinical significance. A total of 164 patients with chronic obstructive pulmonary disease admitted to the outpatient and inpatient departments of our hospital from April 2019 to April 2020 were selected as the study objects. Patients were divided into acute exacerbation group $(n=78)$ and remission group $(n=52)$ according to relevant criteria in chronic obstructive pulmonary disease. There was no significant difference in gender, age, body mass index and other general data among the three groups $(p>0.05)$, showing comparability. The bone mineral density of femoral neck, lumbar spine and Ward's triangle in the three groups had statistical significance $(\mathbf{p}<\mathbf{0 . 0 5})$. The bone metabolism indexes of beta-isomerized C-terminal telopeptides, procollagen type I $\mathrm{N}$ propeptide and osteocalcin in the acute exacerbation group were significantly higher than those in the remission group, the levels of 25 -hydroxyvitamin D3 in the acute exacerbation group were lower than those in the remission group and the differences had statistical significance $(\mathbf{p}<\mathbf{0 . 0 5})$. Pearson correlation analysis showed that bone mineral density of chronic obstructive pulmonary disease patients was negatively correlated with beta-isomerized C-terminal telopeptides, procollagen type I $\mathrm{N}$ propeptide, osteocalcin and positively correlated with 25-hydroxyvitamin D3 $(p<0.05)$. According to Pearson correlation analysis, there was a positive correlation between bone mineral density and 25-hydroxyvitamin $\mathrm{D3}$ in patients with chronic obstructive pulmonary disease $(\mathbf{p}<0.05)$, which was consistent. In conclusion, chronic obstructive pulmonary disease secondary osteoporosis is a high conversion type and bone mineral density and bone metabolism marker levels can effectively predict chronic obstructive pulmonary disease secondary osteoporosis.
\end{abstract}

Key words: Chronic obstructive pulmonary disease, bone mineral density, osteoporosis, bone metabolism indexes

Chronic obstructive pulmonary disease (COPD) is a common and multiple disease characterized by airflow limitation $^{[1]}$. In China, COPD is the most common cause of chronic pulmonary heart disease and chronic respiratory failure, accounting for about $80 \%$ of the total cases ${ }^{[2]}$. The social and economic burden of COPD is enormous. According to the World Health Organization (WHO), COPD will account for $5^{\text {th }}$ place in the world economic burden of disease by 2020. As a result of progressive decline of lung function, a series of extra pulmonary reactions, such as reduction of body mass, malnutrition, osteoporosis, damage to the nervous system and cardiovascular system, have been reported. Osteoporosis is a known major complication of $\mathrm{COPD}^{[3]}$. When COPD is secondary to osteoporosis, the patient's physical condition will deteriorate and the risk of fracture will increase, which will seriously affect the patient's labor force and quality of life. Bone is

*Address for correspondence

E-mail: wuxq515@126.com metabolically active tissue, bone metabolism markers can reflect bone metabolism status, predict fracture risk $^{[4]}$ and provide evidence for standardized diagnosis and treatment of osteoporosis. This study compared the levels of bone metabolism markers in patients with COPD admitted to the outpatient department and the inpatient department of our hospital and evaluated the changes of bone metabolism indexes in patients with COPD and their clinical significance. A total of 164 patients with COPD admitted to the outpatient and inpatient departments of our hospital from April 2019 to April 2020 were selected as the study objects. Patients were divided into acute exacerbation group $(n=78)$ and remission group $(n=52)$ according to relevant criteria in COPD. Diagnosis and treatment guidelines: Inclusion criteria-Diagnosis criteria for COPD exacerbation stage and remission stage in "Global Initiative for Chronic Obstructive Pulmonary Disease in 2017"[]]; Age 18-82 
y; Voluntary cooperation in various examinations; Informed consent of patients. Exclusion criteriaComplicated with hepatic and renal dysfunction; Complicated with endocrine and metabolic diseases such as thyroid or hyperthyroidism; Patients with rheumatic arthritis, osteomalacia, multiple myeloma, fracture, multiple sclerosis and Paget's disease of bone affecting bone metabolism; Continuous use of oral calcium, bisphosphonates, vitamin D3 or other drugs causing secondary changes in bone metabolism. 50 health examiners from the same physical examination center were recruited as the healthy group. There was no significant difference in gender, age, body mass index (BMI) and other general data among the three groups ( $p>0.05)$, showing comparability. See Table 1 . The study was approved by the Ethics Committee of our hospital and the patients/family members signed the informed consent form. LEXXOS dual-energy X-ray absorptiometry (DMS, France) was used to measure the bone mineral density (BMD) of three groups, the proximal left femur (including femoral neck, Ward's triangle) and lumbar spine (L1-L4). According to the WHO, a T-value $\geq-1.0$ standard deviation (SD) indicates normal bone mass; a -2.5 $\mathrm{SD}<\mathrm{T}$-value $<-1.0 \mathrm{SD}$ indicates osteopenia; and a T-value $\leq-2.5$ SD indicates osteoporosis. All study objects were fasted for more than $12 \mathrm{~h}$. On the next day, $5 \mathrm{ml}$ of fasting venous blood was collected in the morning and placed in a test tube. The supernatant was collected by centrifugation at $3000 \mathrm{r} / \mathrm{min}$ for $10 \mathrm{~min}$ and placed in the ultra-low temperature refrigerator for detection. The enzyme-linked immunosorbent assay (ELISA) was used to determine beta-isomerized C-terminal telopeptides $(\beta$-CTX), serum 25-hydroxyvitamin $\quad \mathrm{D}_{3} \quad\left(25-(\mathrm{OH}) \quad \mathrm{D}_{3}\right), \quad \mathrm{N}$-terminal osteocalcin (N-MID) of type I collagen. The electrochemiluminescence method was used to determine N-terminal procollagen N-terminal propeptide (P1NP). All the test kits were purchased from Shanghai Meilian Biology Co., Ltd. The above operations were performed in strict accordance with the instructions for use. Statistical package for the social sciences (SPSS) 22.0 statistical software was used for data analysis in this study. Normal distribution test was performed for measurement data. Mean \pm standard deviation ( $\overline{\mathrm{x}} \pm \mathrm{s})$ was used for normal distribution. Independent sample $t$ test was used for comparison between two groups and analysis of variance was used for multiple groups. [n (\%)] was used for counting data and chi-square test was used. $\alpha=0.05, \mathrm{p}<0.05$ was used for statistical difference. The BMD of femoral neck, lumbar spine and Ward's triangle in the three groups had statistical significance $(p<0.05)$; the BMD of femoral neck, lumbar spine and Ward's triangle in the acute exacerbation group and remission group was higher than that of the healthy control group and the differences had statistical significance $(p<0.05)$; the BMD of femoral neck, lumbar spine and Ward's triangle in the acute exacerbation group was higher than that of the remission group and the differences had statistical significance $(\mathrm{p}<0.05)$. See Table 2 . The bone metabolism indexes of $\beta$-CTX, procollagen type I $\mathrm{N}$ propeptide (PINP), osteocalcin (OC) and 25-(OH) $\mathrm{D}_{3}$ of three groups were compared, with statistical significance $(p<0.05)$; the bone metabolism indexes of $\beta$-CTX, PINP and OC in the acute exacerbation group and the remission group were significantly higher than those in the healthy control group, the levels of $25-(\mathrm{OH}) \mathrm{D}_{3}$ in the acute exacerbation group and the remission group were significantly lower than those in the healthy control group, with statistically significance difference $(p<0.05)$; the bone metabolism indexes of $\beta$-CTX, PINP and OC in the acute exacerbation group were significantly higher than those in the remission group, the levels of $25-(\mathrm{OH}) \mathrm{D}_{3}$ in the acute exacerbation group were lower than those in the remission group and the differences had statistical significance $(p<0.05)$. See Table 3. Pearson correlation analysis showed that BMD of COPD patients was negatively correlated with $\beta$-CTX, PINP, OC and positively correlated with 25$(\mathrm{OH}) \mathrm{D}(\mathrm{p}<0.05)$. See Table 4. COPD is a chronic respiratory disease with high incidence rate, disability rate and case fatality rate in clinic. In recent years, the study showed that ${ }^{[6]}$ the prevalence rate of COPD gradually increased. Studies ${ }^{[7]}$ have shown that the prevalence of osteoporosis in patients with COPD is significantly higher than that in healthy people of the same age, with approximately $36 \%$ to $60 \%$ of patients with COPD having osteoporosis. Because the pathogenesis of osteoporosis is concealed and the symptoms are not obvious, they are usually found when the risk event occurs or the disease is serious and therefore, the optimal treatment period is often missed, and the therapeutic effect and prognosis are poor. Bone turnover markers can make up for the deficiency of bone mineral density assessment and can dynamically and continuously detect the process of measuring change in human body. Therefore, the detection and analysis of markers of osteoporosis bone metabolism in patients with COPD is important for early screening, diagnosis and treatment, as well as prognosis of osteoporosis. In this study, the bone mineral density 
values of each site of patients with COPD in the acute exacerbation group and the remission group were significantly lower than those in the control group, indicating that patients with COPD were more susceptible to osteoporosis than the healthy control group, which was similar to the study results of Luo et al..$^{[8]}$, Shen et al. ${ }^{[9]}$ and Li et al. ${ }^{[10]}$. By comparing the bone mineral density values of femoral neck, lumbar spine and Ward's triangle of patients with COPD in acute exacerbation group, the remission group and the healthy control group, the results showed that the bone mineral density values of femoral neck, lumbar spine and Ward's triangle of patients with COPD in the acute exacerbation group and the remission group were significantly higher than those in the healthy control group ( $\mathrm{p}<0.05$ ), suggesting the risk of osteoporosis in patients with COPD. Looker used multivariate regression analysis to evaluate the relationship between COPD and femoral neck bone mineral density and the effect of femoral neck bone mineral density on death of patients with $\mathrm{COPD}^{[11]}$. The results showed that for each unit decrease in bone density, the risk of death of patients with COPD was increased by $68 \%$, suggesting that the risk of osteoporosis was higher in patients with COPD. The understanding of osteoporosis should be strengthened in clinical practice, and bone mineral density should be monitored in early patients. Bone metabolism formation and absorption in normal body are generally in relatively dynamic balance. Bone metabolism markers include two parts: bone formation markers and bone resorption markers. The measurement of bone metabolism indexes can timely and effectively reflect the rate of bone formation and bone absorption and realize the process of measuring the change of osteoporosis in human body ${ }^{[12,13]}$. The results of this study showed that the bone metabolism indexes of $\beta$-CTX, PINP and OC of patients with COPD in the acute exacerbation group and the remission group were significantly higher than those in the healthy control group, and the levels of 25-(OH) D3 in the acute exacerbation group and the remission group were significantly lower than those in the healthy control group, with statistically significant differences $(\mathrm{p}<0.05)$; it is suggested that osteoporosis in patients with COPD belongs to high turnover type, which may be caused by the imbalance of bone formation and resorption. $\beta$-CTX is an important marker of bone resorption that effectively reflects the osteoclast status and in patients with COPD who have secondary osteoporosis, increases in osteoclast activity as bone resorption increases, leading to increases in $\beta$-CTX levels ${ }^{[14]}$. P1NP is an important marker of bone formation that can objectively reflect osteoblastic status and in patients with secondary osteoporosis, the level of P1NP detection increases when osteoblast activity increases ${ }^{[15]}$. The relatively stable N-MID structure promotes osteoblast formation and effectively reflects osteoblast activity status and osteocyte turnover rate, with a dynamic imbalance in bone metabolism and an increase in N-MID levels when secondary to osteoporosis in patients with $\mathrm{COPD}^{[16]}$. The bone metabolism indexes have high sensitivity, specificity and time-effect, and are safe and non-invasive. It is an important reference index for clinical use in screening and diagnosis and treatment of osteoporosis. 25-(OH) $\mathrm{D}_{3}$ is currently a clinically accepted and widely used index for the assessment of vitamin D status in humans. Data from a number of clinical studies ${ }^{[17]}$ have shown that vitamin D is closely related to bone metabolism in humans, is involved in calcium and phosphorus metabolism in humans and is involved in bone formation; therefore, adequate vitamin D supplementation is the basis for maintaining bone health in humans. Studies have shown that decreasing levels of 25-(OH) $\mathrm{D}_{3}$ cause changes in human blood calcium concentrations and induce an imbalance in bone turnover that disrupts bone microarchitecture ${ }^{[18]}$. In other studies, 25-(OH) D3 levels were positively correlated with BMD performance and 25-(OH) $\mathrm{D}_{3}$ levels were significantly lower in osteoporotic patients than in healthy individuals. According to Pearson correlation analysis, there was a positive correlation between BMD and 25-(OH) $\mathrm{D}_{3}$ in patients with COPD $(p<0.05)$, which was consistent with the above results. In conclusion, COPD secondary osteoporosis is a highconversion type and bone mineral density and bone metabolism marker levels can effectively predict COPD secondary osteoporosis.

\section{TABLE 1: COMPARISON OF GENERAL DATA OF PATIENTS IN THE THREE GROUPS}

\begin{tabular}{|c|c|c|c|c|}
\hline Group & Number of cases & Gender (M/F) & Age (y old) & $\mathrm{BMI}\left(\mathrm{Kg} / \mathrm{m}^{2}\right)$ \\
\hline Acute exacerbation group & 78 & $46 / 32$ & $58.94 \pm 10.56$ & $22.78 \pm 2.26$ \\
\hline Remission group & 54 & $33 / 21$ & $60.07 \pm 10.48$ & $23.33 \pm 2.14$ \\
\hline Healthy control group & 50 & $30 / 20$ & $59.38 \pm 9.97$ & $22.64 \pm 2.45$ \\
\hline $\mathrm{F} / \chi^{2}$ & & 0.061 & 0.189 & 1.394 \\
\hline $\mathrm{p}$ & & 0.97 & 0.828 & 0.251 \\
\hline
\end{tabular}


www.ijpsonline.com

TABLE 2: BONE DENSITY MEASUREMENT RESULTS OF THREE GROUPS $(\mathrm{g} / \mathrm{cm})$

\begin{tabular}{lcccc}
\hline Group & Number of cases & Femoral neck & Lumbar spine (L1-L4) & Ward's triangle \\
\hline Acute exacerbation & 78 & $0.53 \pm 0.07$ & $0.62 \pm 0.10$ & $0.57 \pm 0.06$ \\
group & 54 & $0.72 \pm 0.13$ & $0.83 \pm 0.38$ & $0.65 \pm 0.09$ \\
Remission group & 50 & $0.94 \pm 0.14$ & $1.02 \pm 0.15$ & $0.80 \pm 0.08$ \\
Healthy control group & & 206.6 & 46.83 & 141.7 \\
F & & $<0.001$ & $<0.001$ & $<0.001$ \\
p & & &
\end{tabular}

TABLE 3: COMPARISON OF BONE METABOLISM INDEXES IN THREE GROUPS

\begin{tabular}{|c|c|c|c|c|c|}
\hline Group & Number of cases & B-CTX (ng/ml) & PINP (ng/ml) & $\mathrm{N}-\mathrm{MID}(\mathrm{ng} / \mathrm{ml})$ & 25-(OH) D3 (ng/ml) \\
\hline Acute exacerbation & 78 & $0.70 \pm 0.43$ & $61.08 \pm 18.13$ & $20.11 \pm 1.90$ & $8.67 \pm 3.43$ \\
\hline Remission group & 54 & $0.57 \pm 0.28$ & $50.24 \pm 17.66$ & $17.80 \pm 1.58$ & $12.77 \pm 4.75$ \\
\hline $\begin{array}{l}\text { Healthy control } \\
\text { group }\end{array}$ & 50 & $0.42 \pm 0.19$ & $39.17 \pm 14.63$ & $8.24 \pm 1.45$ & $17.45 \pm 6.29$ \\
\hline $\mathrm{F}$ & & $\begin{array}{c}10.68 \\
<0.001 \\
\end{array}$ & $\begin{array}{r}25.36 \\
<0.001 \\
\end{array}$ & $\begin{array}{r}652.5 \\
<0.001 \\
\end{array}$ & $\begin{array}{c}52.41 \\
<0.001 \\
\end{array}$ \\
\hline
\end{tabular}

TABLE 4: CORRELATION ANALYSIS BETWEEN BMD AND BONE METABOLISM INDEXES OF THE ACUTE EXACERBATION GROUP OF PATIENTS WITH COPD

\begin{tabular}{lcc}
\hline Detection indexes & $r$ & $p$ \\
\hline B-CTX & 0.764 & $<0.001$ \\
PINP & 0.087 & 0.002 \\
OC & 0.166 & 0.006 \\
25- $(\mathrm{OH}) \mathrm{D}_{3}$ & 0.151 & $<0.001$ \\
\hline
\end{tabular}

\section{Conflict of interests:}

The authors declared no conflicts of interest.

\section{REFERENCES}

1. Lin J. Prevention and treatment strategy of osteoporosis secondary to chronic obstructive pulmonary disease. Chin J Tuberc Respir Dis 2015;38(8):617-9.

2. Yang M, He F. Study on the changes of disease activity and bone metabolism markers in patients with chronic obstructive pulmonary disease. Chin J Def Med 2017;31(11):726-9.

3. Kang H, Zhou H, Wei B. Retrospective analysis of bone metabolism markers in 135 patients with spinal cord injury. Chin J Rehabilitation Theor Pract 2021;27(2):156-63.

4. Wang K, Guo S, Feng J. Research Progress on Common Complications of Traditional Chinese Medicine in the Treatment of Chronic Obstructive Pulmonary Disease. West Tradit Chin Med 2021;34(3):149-52.

5. Qin J, Chen C, Shao Y. Clinical Analysis of Bone Mineral Density and Biochemical Indicators of Bone Metabolism in Elderly Male Patients with Chronic Obstructive Pulmonary Disease. Chin J Pulm Dis 2017;10(4):468-70.

6. Zhang Y, Zhao J, Ma L. Etiology of pain caused by acute exacerbation of chronic obstructive pulmonary disease. Chin J Respir Crit Care 2020;19(6):603-5.

7. Wang J, Yang Y, She Q. Analysis of clinical features and related factors in acute exacerbation of chronic obstructive pulmonary disease. Hebei Med J 2017;39(2): 264-5.

8. Luo Q, Zheng W, Xiang Y. Clinical significance of bone mineral density and biochemical index of bone metabolism in patients with COPD. J Bingtuan Med 2019;3:13-5.

9. Shen Q, Cheng Y, Dai L. Changes of bone mineral density and bone metabolism markers in elderly male patients with chronic obstructive pulmonary disease. Chin Med 2019;14(2):218-21.

10. Li S. Clinical analysis of bone mineral density, bone metabolism and biochemical indexes in elderly male patients with chronic obstructive pulmonary disease. Mod J Integr Tradit Chin West Med 2015;24(13):1422-4.
11. Gu L, Zhou M. Retrospective study on changes of biochemical markers of bone metabolism in patients with spinal cord injury. Chin J Rehabil Med 2017;32(12):1356-60.

12. Tan R, Meng Q, Zhang M. Correlation analysis between osteoporotic fracture and bone metabolism markers. Label Immunoassay Clin Med 2020;27(10):1654-8.

13. Zhao G, Luo C, Liu T. Study on P1NP and $\beta$-CTX in patients after cervical spinal cord injury. Chin J Osteoporos 2017;23(12):1617-20.

14. Zhao M. Changes of 25-hydroxyvitamin $\mathrm{D}_{3}$ and bone metabolic parameters and their correlation with bone mineral density in elderly patients with osteoporosis. Naval Med J 2020;41(6):721-3.

15. Li Y, Hou L, Wang C. Bone metabolism markers and their application in the diagnosis of osteoporosis. Chin Med J 2020;17(28):44-7.

16. Yang P, Li X. Diagnostic value of serum $25(\mathrm{OH}) \mathrm{D}$ level in acute exacerbation of chronic obstructive pulmonary disease. Med Inform 2021;34(3):63-7.

17. Sun C, Li Z, Lu Q. Analysis of serum $25(\mathrm{OH})$ D concentration and bone metabolism index levels in patients with type 2 diabetes. Chin J Metall Ind Med 2019;36(3):254-5.

18. Li J, Yu R, Mao J. Correlation between serum 25-(OH) $\mathrm{D}_{3}$ level and lung function and immune function in patients with chronic obstructive pulmonary disease. J Clin Pulm Med 2018;23(2):266-9.

This is an open access article distributed under the terms of the Creative Commons Attribution-NonCommercial-ShareAlike 3.0 License, which allows others to remix, tweak, and build upon the work non-commercially, as long as the author is credited and the new creations are licensed under the identical terms

This article was originally published in a special issue, "Diagnostic and Therapeutic Advances in Biomedical Research and Pharmaceutical Sciences"

Indian J Pharm Sci 2021:83(5) spl issue "24-27" 